\title{
On the Asymptotic Behavior of the Solutions of Some Third and Fourth Order Non-Autonomous Differential Equations
}

By

\author{
Tadayuki HARA*
}

\section{Introduction}

The purpose of this paper is to investigate the asymptotic behavior of the solutions of non-autonomous differential equations of the form

$(1.2) \quad \dddot{x}+a(t) \ddot{x}+b(t) \dot{x}+c(t) h(x)=p(t, x, \dot{x}, \ddot{x})$,

$$
\begin{aligned}
& \dddot{x}+a(t) f(x, \dot{x}) \ddot{x}+b(t) g(x, \dot{x}) \dot{x}+c(t) h(x)=p(t, x, \dot{x}, \ddot{x}), \\
& \dddot{x}+a(t) f(\ddot{x}) \dddot{x}+b(t) \phi(\dot{x}, \ddot{x})+c(t) g(\dot{x})+d(t) h(x)=p(t, x, \dot{x}, \ddot{x}, \dddot{x})
\end{aligned}
$$

where functions appeared in the equations are real valued. The dots indicate differentiation with respect to $t$ and all solutions considered are assumed to be real.

The problem is to give conditions to ensure that all solutions of (1.1), (1.2), (1.3) and (1.4) tend to zero as $t \rightarrow \infty$. This problem has received a considerable amount of attention during the past twenty years, particulary when equations are autonomous. Many of these results are summarized in [14].

In $[17] \mathrm{K}$. E. Swick considered the behavior as $t \rightarrow \infty$ of solutions of the differential equations

$$
\dddot{x}+a \ddot{x}+g(x) \dot{x}+h(x)=e(t),
$$

Communicated by S. Matsuura, June 15, 1973.

* Department of Applied Mathematics, Faculty of Engineering Science, Osaka University, Osaka. 


$$
\dddot{x}+p(t) \ddot{x}+q(t) g(\dot{x})+h(x)=e(t)
$$

where $a$ is a positive constant. In [16] he also considered the asymptotic stability in the large of the trivial solution of the equations

$$
\begin{aligned}
& \dddot{x}+p(t) \ddot{x}+q(t) g(\dot{x})+r(t) h(x)=0, \\
& \dddot{x}+f(x, \dot{x}, t) \ddot{x}+q(t) g(\dot{x})+r(t) h(x)=0 .
\end{aligned}
$$

In [6] the author established the conditions under which all solutions of the non-autonomous equations $(1.1) \sim(1.3)$ tend to zero as $t \rightarrow \infty$.

In this paper we obtain the conditions weaker than that obtained in [6].

Recently the author $([9])$ studied the asymptotic behavior of solutions of

$$
\dddot{x}+a(t) f(x, \dot{x}, \ddot{x}) \ddot{x}+b(t) g(x, \dot{x})+c(t) h(x)=p(t, x, \dot{x}, \ddot{x})
$$

under the condition that

$$
\frac{h(x)}{x} \geqq \delta>0 \quad(x \neq 0) .
$$

But here we consider the equations (1.3) and (1.4) under the weaker condition that

$$
H(x) \equiv \int_{0}^{x} h(\xi) d \xi \rightarrow \infty \quad \text { as }|x| \rightarrow \infty .
$$

In [7] the author also investigated the asymptotic behavior of the solutions of the equation

$$
\dddot{x}+f(\ddot{x}) \dddot{x}+\phi(\dot{x}, \ddot{x})+g(\dot{x})+h(x)=p(t, x, \dot{x}, \ddot{x}, \dddot{x}) .
$$

This time we study the non-autonomous equation (1.4). The results obtained here contains the author's result in [7].

The main tools used in this work are Liapunov functions and the generalized Yoshizawa's Theorem ([21; Theorem 14.2]).

The author whishes to express his appreciation to Dr. M. Yamamoto of Osaka University for his invaluable advice and warm encouragement. 


\section{Assumptions and Statements of the Results}

Theorems 1 and 2 are concerned with the differential equation (1.3). We assume the following assumptions on the functions appeared in (1.3).

\section{Assumptions for Theorems 1 and 2.}

$\left(a_{1}\right) a(t), b(t), c(t)$ are positive and continuously differentiable functions in $I=[0, \infty)$.

$\left(a_{2}\right) \quad p(t, x, y, z)$ is continuous in $I \times R^{3}$.

$\left(a_{3}\right) \quad h(x)$ is continuously differentiable for all $x \in R^{1}$.

$\left(a_{4}\right) f(x, y), f_{x}(x, y), g(x, y)$ and $g_{x}(x, y)$ are continuous for all $(x, y) \in R^{2}$.

Hereafter we use the following notations.

$$
a_{+}^{\prime}(t)=\max \left(a^{\prime}(t), 0\right), \quad a_{-}^{\prime}(t)=\max \left(-a^{\prime}(t), 0\right)
$$

so that $a^{\prime}(t)=a_{+}^{\prime}(t)-a_{-}^{\prime}(t)$. Likewise, we denote

$$
\begin{array}{ll}
b_{+}^{\prime}(t)=\max \left(b^{\prime}(t), 0\right), & b_{-}^{\prime}(t)=\max \left(-b^{\prime}(t), 0\right), \\
c_{+}^{\prime}(t)=\max \left(c^{\prime}(t), 0\right), & c_{-}^{\prime}(t)=\max \left(-c^{\prime}(t), 0\right) .
\end{array}
$$

Theorem 1. Suppose that the assumptions $\left(a_{1}\right) \sim\left(a_{4}\right)$ hold and the following conditions are satisfied:

(i ) $\quad A \geqq a(t) \geqq a_{0}>0, \quad B \geqq b(t) \geqq b_{0}>0, \quad C \geqq c(t) \geqq c_{0}>0 \quad$ for $t \in I$,

(ii) $f_{1} \geqq f(x, y) \geqq f_{0}>0, \quad y f_{x}(x, y) \leqq 0 \quad$ for all $(x, y) \in R^{2}$,

(iii) $g_{1} \geqq g(x, y) \geqq g_{0}>0, \quad y g_{x}(x, y) \leqq 0 \quad$ for all $(x, y) \in R^{2}$,

(iv) $x h(x)>0 \quad(x \neq 0), \quad H(x) \equiv \int_{0}^{x} h(\xi) d \xi \rightarrow \infty \quad a s|x| \rightarrow \infty$,

(v) $\frac{a_{0} b_{0} f_{0} g_{0}}{C}>h_{1} \geqq h^{\prime}(x)$,

(vi) $\mu\left\{a_{+}^{\prime}(t) f_{1}-a_{-}^{\prime}(t) f_{0}\right\}+\left\{b_{+}^{\prime}(t) g_{1}-b_{-}^{\prime}(t) g_{0}\right\}$

$$
-\frac{1}{\mu} c^{\prime}(t) h_{1}<\mu b_{0} g_{0}-C h_{1}
$$

where $\mu$ is an arbitrarily fixed constant satisfying $\frac{C h_{1}}{b_{0} g_{0}}<\mu<a_{0} f_{0}$, 


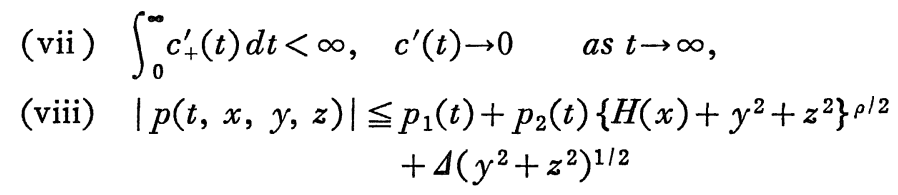

where $\rho, \Delta$ are constants such that $0 \leqq \rho \leqq 1, \Delta \geqq 0$ and $p_{1}(t)$, $p_{2}(t)$ are non-negative continuous functions satisfying,

(ix) $\int_{0}^{\infty} p_{i}(t) d t<\infty \quad(i=1,2)$.

If $\Delta$ is sufficiently small, then every solution $x(t)$ of (1.3) is uniformbounded and satisfies

$$
x(t) \rightarrow 0, \quad \dot{x}(t) \rightarrow 0, \quad \ddot{x}(t) \rightarrow 0 \quad \text { as } t \rightarrow \infty .
$$

As an immediate consequence of Theorem 1, we have the following result on (1.1).

Corollary 1. Suppose that the assumption $\left(a_{1}\right)$ and the conditions (i), (vii) of Theorem 1 hold and in addition the following conditions are satisfied:

(v) ${ }^{\prime} \quad a_{0} b_{0}-C>0$

(vi) $\quad \mu a^{\prime}(t)+b^{\prime}(t)-\frac{1}{\mu} c^{\prime}(t)<\mu b_{0}-C \quad\left(\frac{C}{b_{0}}<\mu<a_{0}\right)$,

$(\mathrm{ix})^{\prime} \quad \int_{0}^{\infty}|p(t)| d t<\infty$.

Then every solution $x(t)$ of (1.1) is uniform-bounded and satisfies

$$
x(t) \rightarrow 0, \quad \dot{x}(t) \rightarrow 0, \quad \ddot{x}(t) \rightarrow 0 \quad \text { as } t \rightarrow \infty .
$$

And also we have the following Corollary 2 concerning the equation (1.2).

Corollary 2. Suppose that the assumptions $\left(a_{1}\right) \sim\left(a_{3}\right)$ and the conditions (i), (iv), (vii) of Theorem 1 hold and the following conditions are satisfied:

$$
\begin{aligned}
& \text { (v) } \quad \frac{a_{0} b_{0}}{C}>h_{1} \geqq h^{\prime}(x), \\
& \text { (vi) } \quad \mu a^{\prime}(t)+b^{\prime}(t)-\frac{1}{\nu} c^{\prime}(t)<\mu b_{0}-C h_{1} \quad\left(\frac{C h_{1}}{b_{0}}<\mu<a_{0}, \quad \nu=\frac{\mu}{h_{1}}\right),
\end{aligned}
$$


(viii) $^{\prime}|p(t, x, y, z)| \leqq p_{1}(t)$,

$(\mathrm{ix})^{\prime} \int_{0}^{\infty} p_{1}(t) d t<\infty$.

Then every solution $x(t)$ of (1.2) is uniform-bounded and satisfies

$$
x(t) \rightarrow 0, \quad \dot{x}(t) \rightarrow 0, \quad \ddot{x}(t) \rightarrow 0 \quad \text { as } t \rightarrow \infty .
$$

Remark. Theorem 1 extends the author's earlier results, that is, Corollaries 1 and 2 coincide with Corollary 1 and Theorem 1 in [6] respectively.

In $[4]$, J.O.C. Ezeilo studied the equation

$$
\ddot{x}+f_{1}(x, \dot{x}) \ddot{x}+f_{2}(\dot{x})+f_{3}(x)=p(t, x, \dot{x}, \ddot{x})
$$

where $p(t, x, y, z)$ satisfies the condition (viii) of our Theorem 1 . He required the boundedness and integrability of the functions $p_{1}(t)$ and $p_{2}(t)$. Here we only assume the integrability of $p_{1}(t)$ and $p_{2}(t)$.

Observe that the condition (v) in Theorem 1 is the usual «generalized Routh-Hurwitz conditions 》.

Theorem 2. Suppose that the assumptions $\left(a_{1}\right) \sim\left(a_{4}\right)$ hold and the following conditions are satisfied:

(i) $\quad A \geqq a(t) \geqq a_{0}>0, \quad B \geqq b(t) \geqq b_{0}>0, \quad C \geqq c(t) \geqq c_{0}>0 \quad$ for $t \in I$,

(ii) $f(x, y) \geqq f_{0}>0, \quad y f_{x}(x, y) \leqq 0 \quad$ for all $(x, y) \in R^{2}$,

(iii) $g(x, y) \geqq g_{0}>0, \quad y g_{x}(x, y) \leqq 0$ for all $(x, y) \in R^{2}$,

(iv) $x h(x)>0 \quad(x \neq 0), \quad H(x) \equiv \int_{0}^{x} h(\xi) d \xi \rightarrow \infty \quad$ as $|x| \rightarrow \infty$,

(v) $\frac{a_{0} b_{0} f_{0} g_{0}}{C}>h_{1} \geqq h^{\prime}(x)$,

(vi) $\int_{0}^{\infty}\left\{a_{+}^{\prime}(t)+b_{+}^{\prime}(t)+\left|c^{\prime}(t)\right|\right\} d t<\infty, \quad c^{\prime}(t) \rightarrow 0$ as $t \rightarrow \infty$,

(vii) $|p(t, x, y, z)| \leqq p_{1}(t)+p_{2}(t)\left\{H(x)+y^{2}+z^{2}\right\}^{\rho / 2}+\Delta\left(y^{2}+z^{2}\right)^{1 / 2}$ where $\rho, \Delta$ are constants such that $0 \leqq \rho \leqq 1, \Delta \geqq 0$ and $p_{1}(t), p_{2}(t)$ are non-negative continuous functions satisfying,

(viii) $\int_{0}^{\infty} p_{i}(t) d t<\infty \quad(i=1,2)$.

If $\Delta$ is sufficiently small, then every solution $x(t)$ of (1.3) is uniform- 
bounded and satisfies

$$
x(t) \rightarrow 0, \quad \dot{x}(t) \rightarrow 0, \quad \ddot{x}(t) \rightarrow 0 \quad \text { as } t \rightarrow \infty .
$$

Remark. In Theorem 2 the functions $f(x, y)$ and $g(x, y)$ are not generally bounded above. Here also we do not need the boundedness of the functions $p_{1}(t)$ and $p_{2}(t)$. Theorem 2 is the extension of the author's earlier result $([6$; Theorem 2$])$.

We turn now to the fourth order differential equation (1.4). We make the following assumptions on the functions appeared in (1.4).

\section{Assumptions for Theorem 3.}

$\left(A_{1}\right) a(t), b(t), c(t)$ and $d(t)$ are positive and continuously differentiable functions in $I=[0, \infty)$.

$\left(A_{2}\right) \quad f(z)$ is continuously differentiable for all $z \in R^{1}$.

$\left(A_{3}\right) \quad \phi(y, z)$ and $\frac{\partial \phi}{\partial y}(y, z)$ are continuous for all $(y, z) \in R^{2}$.

$\left(A_{4}\right) \quad g(y)$ is continuously differentiable for all $y \in R^{1}$.

$\left(A_{5}\right) \quad h(x)$ is continuously differentiable for all $x \in R^{1}$.

$\left(A_{6}\right) \quad p(t, x, y, z, w)$ is continuous in $I \times R^{4}$.

In Theorem 3, the following notations are used:

$$
\begin{gathered}
g_{1}(y)=\frac{g(y)}{y} \quad(y \neq 0), \quad g_{1}(0)=g^{\prime}(0), \\
f_{1}(z)=\frac{1}{z} \int_{0}^{z} f(\xi) d \xi \quad(z \neq 0), \quad f_{1}(0)=f(0) .
\end{gathered}
$$

Theorem 3. Suppose that the assumptions $\left(A_{1}\right) \sim\left(A_{6}\right)$ hold and that there exist positive constants such that
(i ) $\quad A \geqq a(t) \geqq a_{0}>0, \quad B \geqq b(t) \geqq b_{0}>0, \quad C \geqq c(t) \geqq c_{0}>0$, $D \geqq d(t) \geqq d_{0}>0 \quad$ for $t \in I$,
(ii) $f(z) \geqq f_{0}>0 \quad$ for all $z \in R^{1}$,
(iii) $g_{1}(y) \geqq g_{0}>0 \quad$ for all $y \in R^{1}, \quad g(0)=0$,
(iv) $x h(x)>0 \quad(x \neq 0), \quad H(x) \equiv \int_{0}^{x} h(\xi) d \xi \rightarrow \infty \quad$ as $|x| \rightarrow \infty$, 


$$
\begin{aligned}
& h_{0}-\frac{a_{0} f_{0} \delta_{0}}{2 c_{0} g_{0} D} \leqq h^{\prime}(x) \leqq h_{0}, \\
& \text { (v) } \phi_{y}(y, z) \leqq 0, \quad \phi(y, 0)=0 \quad \text { in } R^{2} \text {, } \\
& \text { (vi) } 0 \leqq \frac{\phi(y, z)}{z}-\phi_{0} \leqq \frac{\varepsilon_{0} c_{0}^{3} g_{0}^{3}}{B D^{2} h_{0}^{2}} \quad(z \neq 0) \\
& \text { where } \varepsilon_{0} \text { is a sufficiently small positive constant, } \\
& \text { (vii) } a_{0} b_{0} c_{0} f_{0} \phi_{0} g_{0}-C^{2} g_{0} g^{\prime}(y)-A^{2} D f_{0} h_{0} f(z) \geqq \delta_{0}>0 \\
& \text { for all }(y, z) \in R^{2} \text {, } \\
& \text { (viii) } g^{\prime}(y)-g_{1}(y) \leqq \delta<\frac{2 D h_{0} \delta_{0}}{C a_{0} f_{0} c_{0}^{2} g_{0}^{2}} \text {, } \\
& \text { (ix) } f_{1}(z)-f(z) \leqq \frac{C c_{0} g_{0} \delta}{A a_{0} f_{0} D h_{0}} \text {, } \\
& \text { (x) } \int_{0}^{\infty}\left\{\left|a^{\prime}(t)\right|+b_{+}^{\prime}(t)+\left|c^{\prime}(t)\right|+\left|d^{\prime}(t)\right|\right\} d t<\infty \text {, } \\
& d^{\prime}(t) \rightarrow 0 \quad \text { as } t \rightarrow \infty \text {, where } b_{+}^{\prime}(t)=\max \left(b^{\prime}(t), 0\right), \\
& \text { (xi) }|p(t, x, y, z, w)| \leqq p_{1}(t)+p_{2}(t)\left\{H(x)+y^{2}+z^{2}+w^{2}\right\}^{\rho / 2} \\
& +\Delta\left(y^{2}+z^{2}+w^{2}\right)^{1 / 2} \\
& \text { where } \rho, \Delta \text { are constants such that } 0 \leqq \rho \leqq 1, \Delta \geqq 0 \text { and } p_{1}(t) \text {, } \\
& p_{2}(t) \text { are non-negative continuous functions satisfying, } \\
& \text { (xii) } \int_{0}^{\infty} p_{i}(t) d t<\infty \quad(i=1,2) \text {. }
\end{aligned}
$$

If $\Delta$ is sufficiently small, then every solution $x(t)$ of (1.4) is uniformbounded and satisfies

$$
x(t) \rightarrow 0, \quad \dot{x}(t) \rightarrow 0, \quad \dot{x}(t) \rightarrow 0, \quad \dddot{x}(t) \rightarrow 0 \quad \text { as } t \rightarrow \infty .
$$

Remark. Theorem 3 extends the author's result [7] to the nonautonomous equation (1.4). Theorem 3 also contains the results obtained by J.O.C. Ezeilo [4], M. Harrow [11] and M. A. Asmussen [1]. Note that also we do not require the boundedness of $p_{1}(t)$ and $p_{2}(t)$ here.

\section{Auxiliary Lemmas}

Consider a system of differential equations

$$
\dot{X}=F(t, X)
$$

where $X=\left(x_{1}, \ldots, x_{n}\right)$ and $F(t, X)$ is continuous in $I \times R^{n}(I=[0, \infty))$. 
The following Lemma 1 is well-known ([21]).

Lemma 1. Suppose that there exists a continuously differentiable function $V(t, X)$ defined on $t \in I,\|X\| \geqq R$, where $R$ may be large, which satisfies the following conditions:

(i) $a(\|X\|) \leqq V(t, X) \leqq b(\|X\|)$, where $a(r) \in C I$ (a family of continuous and increasing functions), $a(r) \rightarrow \infty$ as $r \rightarrow \infty$ and $b(r) \in C I$,

(ii) $\dot{V}_{(3.1)}(t, X) \leqq 0$.

Then the solutions of (3.1) are uniform bounded.

Next we consider a system of differential equations

$$
\dot{X}=F(t, X)+G(t, X)
$$

where $F(t, X)$ and $G(t, X)$ are continuous on $I \times Q \quad(I=[0, \infty), Q$ : an open set in $R^{n}$ ). We assume

$$
\|G(t, X)\| \leqq G_{1}(t, X)+G_{2}(X)
$$

where $G_{1}(t, X)$ is non-negative continuous on $I \times Q$ and $\int_{0}^{t} G_{1}(s, X) d s$ is bounded for all $t$ whenever $X$ belongs to any compact subset of $Q$, and $G_{2}(X)$ is non-negative continuous in $Q$.

The following Lemma is a simple extension of the well-known result obtained by $\mathrm{T}$. Yoshizawa [21; Theorem 14.2].

Lemma 2. Suppose that there exists a non-negative continuously differentiable function $V(t, X)$ on $I \times Q$ such that $\dot{V}_{(3.2)}(t, X) \leqq-W(X)$, where $W(X)$ is positive definite with respect to a closed set $\Omega$ in the space $Q$. Moreover, suppose that $F(t, X)$ of the system (3.1) is bounded for all $t$ when $X$ belongs to an arbitrary compact set in $Q$ and that $F(t, X)$ satisfies the following two conditions with respect to $\Omega$ :

(a) $F(t, X)$ tends to a function $H(X)$ for $X \in \Omega$ as $t \rightarrow \infty$, and on any compact set in $\Omega$ this convergence is uniform.

(b) Corresponding to each $\varepsilon>0$ and each $Y \in \Omega$, there exist a $\delta(\varepsilon, Y)$ and a $T(\varepsilon, Y)$ such that if $\|X-Y\|<\delta(\varepsilon, Y)$ and $t \geqq T(\varepsilon, Y)$, we have $\|F(t, X)-F(t, Y)\|<\varepsilon$. And suppose that 
(c) $G_{2}(X)$ is positive definite with respect to a closed set $\Omega$ in the space $Q$.

Then, every bounded solution of (3.2) approaches the largest semi-invariant set of the system $\dot{X}=H(X)$ contained in $\Omega$ as $t \rightarrow \infty$.

Proof of Lemma 2. The proof runs analogously as the original proof $[21 ;$ p. $52 \sim$ p. 61$]$ using the fact that for any $\lambda>0$

$$
\int_{t}^{t+\lambda} G_{2}(x(s)) d s \rightarrow 0 \quad \text { as } t \rightarrow \infty
$$

whenever $x(t)$ approaches to $\Omega$ as $t \rightarrow \infty$ e.g.

\section{Proof of Theorem 1}

In this section it will be assumed that $X=(x, y, z)$ and $\|X\|$ $=\sqrt{x^{2}+y^{2}+z^{2}}$.

We consider, in place of (1.3), the equivalent system

$$
\text { (4.1) }\left\{\begin{array}{l}
\dot{x}=y \\
\dot{y}=z \\
\dot{z}=-a(t) f(x, y) z-b(t) g(x, y) y-c(t) h(x)+p(t, x, y, z) .
\end{array}\right.
$$

Consider the Liapunov function defined by

$$
\begin{aligned}
V_{0}(t, x, y, z)= & \mu c(t) H(x)+c(t) h(x) y+b(t) \int_{0}^{y} g(x, \eta) \eta d \eta \\
& +\mu a(t) \int_{0}^{y} f(x, \eta) \eta d \eta+\mu y z+\frac{1}{2} z^{2}+k
\end{aligned}
$$

where $k$ is a non-negative constant to be determined later in the proof.

Let $\nu=\frac{\mu}{h_{1}}$, then we have

$$
\begin{aligned}
V_{0}= & \frac{1}{2} \mu c(t)\left\{2 H(x)+\frac{2}{\mu} h(x) y+\frac{1}{\mu \nu} y^{2}\right\} \\
& +\frac{1}{\nu} \int_{0}^{y}\{\nu b(t) g(x, \eta)-c(t)\} \eta d \eta
\end{aligned}
$$




$$
+\mu \int_{0}^{y}\{a(t) f(x, \eta)-\mu\} \eta d \eta+\frac{1}{2}(z+\mu y)^{2}+k .
$$

Since $h_{1} \geqq h^{\prime}(x)$, we have $2 h_{1} H(x) \geqq h^{2}(x)$.

Then it follows

$$
|y| \sqrt{2 h_{1} H(x)} \geqq h(x) y \geqq-|y| \sqrt{2 h_{1} H(x)}
$$

and

$$
\begin{aligned}
\left(\sqrt{2 H(x)}+\frac{|y|}{\nu \sqrt{h_{1}}}\right)^{2} & \geqq\left\{2 H(x)+\frac{2}{\nu h_{1}} h(x) y+\frac{1}{\nu^{2} h_{1}} y^{2}\right\} \\
& \geqq\left(\sqrt{2 H(x)}-\frac{|y|}{\nu \sqrt{h_{1}}}\right)^{2} .
\end{aligned}
$$

The left hand side of $(4.3)=2 \delta_{0} H(x)+\left(\sqrt{2\left(1-\delta_{0}\right) H(x)}+\frac{|y|}{\nu \sqrt{\left(1-\delta_{0}\right) h_{1}}}\right)^{2}$

$$
-\frac{\delta_{0}}{\left(1-\delta_{0}\right) \nu^{2} h_{1}} y^{2}
$$

The right hand side of $(4.3)=2 \delta_{0} H(x)+\left(\sqrt{2\left(1-\delta_{0}\right) H(x)}-\frac{|y|}{\nu \sqrt{\left(1-\delta_{0}\right) h_{1}}}\right)^{2}$

$$
-\frac{\delta_{0}}{\left(1-\delta_{0}\right) \nu^{2} h_{1}} y^{2}
$$

Hence we have

$$
\begin{aligned}
\mu c(t) \delta_{0} H(x) & +\frac{1}{2} \mu c(t)\left\{\sqrt{2\left(1-\delta_{0}\right) H(x)}+\frac{|y|}{\nu \sqrt{\left(1-\delta_{0}\right) h_{1}}}\right\}^{2} \\
& -\frac{1}{2} c(t) \frac{\delta_{0}}{\left(1-\delta_{0}\right) \nu} y^{2}+\frac{1}{\nu} \int_{0}^{y}\{\nu b(t) g(x, \eta)-c(t)\} \eta d \eta \\
& +\mu \int_{0}^{y}\{a(t) f(x, \eta)-\mu\} \eta d \eta+\frac{1}{2}(z+\mu y)^{2}+k \\
\geqq & V_{0} \geqq \mu c(t) \delta_{0} H(x)+\frac{1}{2} \mu c(t)\left\{\sqrt{2\left(1-\delta_{0}\right) H(x)}-\frac{|y|}{\nu \sqrt{\left(1-\delta_{0}\right) h_{1}}}\right\}^{2} \\
& -\frac{1}{2} c(t) \frac{\delta_{0}}{\left(1-\delta_{0}\right) \nu} y^{2}+\frac{1}{\nu} \int_{0}^{y}\{\nu b(t) g(x, \eta)-c(t)\} \eta d \eta
\end{aligned}
$$




$$
+\mu \int_{0}^{y}\{a(t) f(x, \eta)-\mu\} \eta d \eta+\frac{1}{2}(z+\mu y)^{2}+k .
$$

If we take $\delta_{0}$ as $1-\frac{C}{\nu b_{0} g_{0}}>\delta_{0}>0$, we have

$$
\begin{aligned}
V_{0} \geqq & \mu c(t) \delta_{0} H(x)+\frac{1}{\nu} \int_{0}^{y}\left\{\nu b(t) g(x, \eta)-\frac{c(t)}{1-\delta_{0}}\right\} \eta d \eta \\
& +\mu \int_{0}^{y}\{a(t) f(x, \eta)-\mu\} \eta d \eta+\frac{1}{2}(z+\mu y)^{2}+k \\
= & \mu c(t) \delta_{0} H(x)+\frac{1}{\nu} \int_{0}^{y}\left\{\nu b(t) g(x, \eta)-\frac{c(t)}{1-\delta_{0}}\right\} \eta d \eta \\
& +\mu \int_{0}^{y}\left\{a(t) f(x, \eta)-\mu-\mu \delta_{1}\right\} \eta d \eta \\
& +\frac{1}{2}\left(1+\delta_{1}\right) \mu^{2}\left\{y+\frac{z}{\left(1+\delta_{1}\right) \mu}\right\}^{2}+\frac{\delta_{1}}{2\left(1+\delta_{1}\right)} z^{2}+k .
\end{aligned}
$$

Here we take $\delta_{1}$ as $\frac{a_{0} f_{0}-\mu}{\mu}>\delta_{1}>0$, then

$$
\begin{aligned}
V_{0} \geqq & \mu c(t) \delta_{0} H(x)+\frac{1}{\nu} \int_{0}^{y}\left\{\nu b(t) g(x, \eta)-\frac{c(t)}{1-\delta_{0}}\right\} \eta d \eta \\
& +\mu \int_{0}^{y}\left\{a(t) f(x, \eta)-\mu-\mu \delta_{1}\right\} \eta d \eta+\frac{\delta_{1}}{2\left(1+\delta_{1}\right)} z^{2}+k
\end{aligned}
$$

and we can find a positive number $D_{1}$ such that

$$
V_{0}(t, x, y, z) \geqq D_{1}\left\{H(x)+y^{2}+z^{2}+k\right\} \text {. }
$$

It is easy to see that there exist two continuous functions $w_{1}(r)$ and $w_{2}(r)$ such that

$$
w_{1}(\|X\|) \leqq V_{0}(t, x, y, z) \leqq w_{2}(\|X\|)
$$

for all $X \in R^{3}$ and $t \in I$ where $w_{1}(r) \in C I P$ (a family of continuous increasing positive definite functions), $w_{1}(r) \rightarrow \infty$ as $r \rightarrow \infty$ and $w_{2}(r) \in C I$.

Along any solution $(x(t), y(t), z(t))$ of (4.1), we have

$$
\dot{V}_{0(4.1)}=-\left[\mu b(t) g(x, y)-c(t) h^{\prime}(x)\right] y^{2}-[a(t) f(x, y)-\mu] z^{2}
$$




$$
\begin{aligned}
& +\frac{1}{2} \mu c^{\prime}(t)\left\{2 H(x)+\frac{2}{\mu} h(x) y+\frac{1}{\mu \nu} y^{2}\right\}+b(t) y \int_{0}^{y} g_{x}(x, \eta) \eta d \eta \\
& +\mu a(t) y \int_{0}^{y} f_{x}(x, \eta) \eta d \eta+(\mu y+z) p(t, x, y, z) \\
& +\int_{0}^{y}\left\{\mu a^{\prime}(t) f(x, \eta)+b^{\prime}(t) g(x, \eta)-\frac{1}{\nu} c^{\prime}(t)\right\} \eta d \eta .
\end{aligned}
$$

By the conditions (ii), (iii) and (vi),

$$
\begin{aligned}
\dot{V}_{0(4.1) \leqq} & -\left(\mu b_{0} g_{0}-C h_{1}\right) y^{2}-\left(a_{0} f_{0}-\mu\right) z^{2}+\frac{c_{+}^{\prime}(t)}{c(t)} V_{0} \\
& +\frac{1}{2}\left(\mu b_{0} g_{0}-C h_{1}\right) y^{2}+(1+\mu)(|y|+|z|)|p(t, x, y, z)| \\
\leqq & -\frac{1}{2}\left(\mu b_{0} g_{0}-C h_{1}\right) y^{2}-\left(a_{0} f_{0}-\mu\right) z^{2}+\frac{c_{+}^{\prime}(t)}{c(t)} V_{0} \\
& +\sqrt{2}(1+\mu)\left\{p_{1}(t)+p_{2}(t)\left(H(x)+y^{2}+z^{2}\right)^{\rho / 2}\right\}\left(y^{2}+z^{2}\right)^{1 / 2} \\
& +\sqrt{2} \Delta(1+\mu)\left(y^{2}+z^{2}\right) .
\end{aligned}
$$

Note that

$$
\left(H(x)+y^{2}+z^{2}\right)^{\rho / 2} \leqq 1+\left(H(x)+y^{2}+z^{2}\right)^{1 / 2}
$$

and if we take $\Delta<\min \left\{\frac{\mu b_{0} g_{0}-C h_{1}}{2 \sqrt{2}(1+\mu)}, \frac{a_{0} f_{0}-\mu}{\sqrt{2}(1+\mu)}\right\}$, we can find a positive number $D_{2}$ such that

$$
\begin{aligned}
\dot{V}_{0(4.1)} \leqq & -D_{2}\left(y^{2}+z^{2}\right)+\frac{c_{+}^{\prime}(t)}{c(t)} V_{0} \\
& +\sqrt{2}(1+\mu)\left\{p_{1}(t)+p_{2}(t)\right\}\left(y^{2}+z^{2}\right)^{1 / 2} \\
& +\sqrt{2}(1+\mu) p_{2}(t)\left(H(x)+y^{2}+z^{2}\right)
\end{aligned}
$$

Now we define

$$
V(t, x, y, z)=e^{-\int_{0}^{t} \gamma(s) d s} \cdot V_{0}(t, x, y, z)
$$

where 


$$
\gamma(s)=\frac{c_{+}^{\prime}(s)}{c(s)}+\frac{2 \sqrt{2}(1+\mu)}{D_{1}}\left\{p_{1}(s)+p_{2}(s)\right\}
$$

Then it is easily verified that there exist two continuous functions $\tilde{w}_{1}(r), \tilde{w}_{2}(r)$ satisfying

$$
\tilde{w}_{1}(\|X\|) \leqq V(t, x, y, z) \leqq \tilde{w}_{2}(\|X\|)
$$

for all $X \in R^{3}$ and $t \in I$ where $\tilde{w}_{1}(r) \in C I P, \tilde{w}_{1}(r) \rightarrow \infty$ as $r \rightarrow \infty$ and $\tilde{w}_{2}(r) \in$ CI.

Along any solution $(x(t), y(t), z(t))$ of (4.1) we have

$$
\begin{aligned}
\dot{V}_{(4.1)}= & e^{-\int_{0}^{t} \gamma(s) d s} \cdot\left[\dot{V}_{0(4.1)}-\gamma(t) V_{0}\right] \\
\leqq & e^{-\int_{0}^{t} \gamma(s) d s} \cdot\left[-D_{2}\left(y^{2}+z^{2}\right)+\sqrt{2}(1+\mu)\left\{p_{1}(t)+p_{2}(t)\right\}\left(y^{2}+z^{2}\right)^{1 / 2}\right. \\
& \left.-\sqrt{2}(1+\mu)\left\{p_{1}(t)+p_{2}(t)\right\}\left\{H(x)+y^{2}+z^{2}+2 k\right\}\right] \\
\leqq & e^{-\int_{0}^{t} \gamma(s) d s} \cdot\left[-D_{2}\left(y^{2}+z^{2}\right)\right. \\
& \left.-\sqrt{2}(1+\mu)\left\{p_{1}(t)+p_{2}(t)\right\}\left\{\left(\sqrt{y^{2}+z^{2}}-\frac{1}{2}\right)^{2}-\frac{1}{4}+2 k\right\}\right] .
\end{aligned}
$$

Setting $k \geqq \frac{1}{8}$, we can find a positive number $D_{3}$ such that

$$
\dot{V}_{(4.1)} \leqq-D_{3}\left(y^{2}+z^{2}\right)
$$

From the inequalities (4.10), (4.11) and Lemma 1, we see that all the solutions $(x(t), y(t), z(t))$ of (4.1) are uniform-bounded.

In the system (4.1) we set

$$
F(t, X)=\left(\begin{array}{c}
x \\
y \\
-a(t) f(x, y) z-b(t) g(x, y) y-c(t) h(x)
\end{array}\right),
$$

$$
G(t, X)=\left(\begin{array}{c}
0 \\
0 \\
p(t, x, y, z)
\end{array}\right)
$$


then

$$
\|G(t, X)\| \leqq p_{1}(t)+p_{2}(t)\left\{H(x)+y^{2}+z^{2}\right\}^{\rho / 2}+\Delta\left(y^{2}+z^{2}\right)^{1 / 2} .
$$

Let $G_{1}(t, X)=p_{1}(t)+p_{2}(t)\left\{H(x)+y^{2}+z^{2}\right\}^{\rho / 2}$ and $G_{2}(X)=\Delta\left(y^{2}+z^{2}\right)^{1 / 2}$.

It is clear that $F(t, X)$ and $G_{1}(t, X)$ satisfy the conditions of Lemma 2.

Let $W(X)=D_{3}\left(y^{2}+z^{2}\right)$, then

$$
\dot{V}_{(4.1)}(t, x, y, z) \leqq-W(X)
$$

and $W(X)$ is positive definite with respect to the closed set $\Omega \equiv\{(x, y, z) \mid$ $\left.x \in R^{1}, y=0, z=0\right\}$. It follows that on $\Omega$

$$
F(t, X)=\left(\begin{array}{c}
0 \\
0 \\
-c(t) h(x)
\end{array}\right)
$$

By the condition (i) and (vii), we have $c(t) \rightarrow c_{\infty}$ as $t \rightarrow \infty$ where $0<c_{0} \leqq c_{\infty} \leqq C$. It is also clear that if we take

$$
\tilde{H}(X) \equiv\left(\begin{array}{c}
0 \\
0 \\
-c_{\infty} h(x)
\end{array}\right),
$$

then the conditions (a) and (b) of Lemma 2 are satisfied.

Moreover $G_{2}(X)$ is positive definite with respect to the closed set $\Omega$ and the condition (c) of Lemma 2 is satisfied.

Since all the solutions of (4.1) are bounded, it follows from Lemma 2 that every solution of (4.1) approaches the largest semi-invariant set of $\dot{X}=\tilde{H}(X)$ contained in $\Omega$ as $t \rightarrow \infty$.

From (4.13), $\dot{X}=\tilde{H}(X)$ is the system

$$
\dot{x}=0, \quad \dot{y}=0, \quad \dot{z}=-c_{\infty} h(x)
$$

which has the solutions $x=c_{1}, y=c_{2}, z=c_{3}-c_{\infty} h\left(c_{1}\right)\left(t-t_{0}\right)$. To remain in $\Omega, c_{2}=0$ and $c_{3}-c_{\infty} h\left(c_{1}\right)\left(t-t_{0}\right)=0$ for all $t \geqq t_{0}$ which implies $c_{1}=c_{3}=0$.

Therefore the only solution of $\dot{X}=\tilde{H}(X)$ remaining in $\Omega$ is $X \equiv 0$, that is, the largest semi-invariant set of $\dot{X}=\tilde{H}(X)$ contained in $\Omega$ is the 
point $(0,0,0)$. Then it follows that

$$
x(t) \rightarrow 0, \quad \dot{x}(t) \rightarrow 0, \quad \ddot{x}(t) \rightarrow 0 \text { as } t \rightarrow \infty .
$$

\section{Proof of Theorem 2}

Here we consider the system (4.1) and the Liapunov function (4.2), and denote $X=(x, y, z)$ and $\|X\|=\sqrt{x^{2}+y^{2}+z^{2}}$.

By the same arguments as before we obtain the estimates (4.4), (4.5) and (4.6). Then,

$$
\begin{aligned}
\dot{V}_{0(4.1) \leqq} & -\left(\mu b_{0} g_{0}-C h_{1}\right) y^{2}-\left(a_{0} f_{0}-\mu\right) z^{2}+\frac{\left|c^{\prime}(t)\right|}{c(t)} V_{0} \\
& +\frac{a_{+}^{\prime}(t)}{a(t)} \cdot \mu a(t) \int_{0}^{y} f(x, \eta) \eta d \eta+\frac{b_{+}^{\prime}(t)}{b(t)} \cdot b(t) \int_{0}^{y} g(x, \eta) \eta d \eta \\
& +\frac{\left|c^{\prime}(t)\right|}{\nu} y^{2}+(1+\mu)(|y|+|z|)|p(t, x, y, z)|
\end{aligned}
$$

where $\mu$ is an arbitrarily fixed constant satisfying $\frac{C h_{1}}{b_{0} g_{0}}<\mu<a_{0} f_{0}$.

Note that

$$
\begin{aligned}
\mu a(t) \int_{0}^{y} f(x, \eta) \eta d \eta & =\mu \int_{0}^{y}\left\{a(t) f(x, \eta)-\mu-\mu \delta_{1}\right\} \eta d \eta+\frac{1}{2} \mu^{2}\left(1+\delta_{1}\right) y^{2} \\
& \leqq V_{0}+\frac{1}{2} \mu^{2}\left(1+\delta_{1}\right) y^{2} \\
b(t) \int_{0}^{y} g(x, \eta) \eta d \eta & =\frac{1}{\nu} \int_{0}^{y}\left\{\nu b(t) g(x, \eta)-\frac{c(t)}{1-\delta_{0}}\right\} \eta d \eta+\frac{c(t)}{2 \nu\left(1-\delta_{0}\right)} y^{2} \\
& \leqq V_{0}+\frac{C}{2 \nu\left(1-\delta_{0}\right)} y^{2}
\end{aligned}
$$

where $\delta_{0}, \delta_{1}$ are positive constants determined in the Proof of Theorem 1. Then we have

$$
\begin{aligned}
\dot{V}_{0(4.1)} & \leqq-\left(\mu b_{0} g_{0}-C h_{1}\right) y^{2}-\left(a_{0} f_{0}-\mu\right) z^{2} \\
& +\frac{\left|c^{\prime}(t)\right|}{c(t)} V_{0}+\frac{a_{+}^{\prime}(t)}{a(t)} V_{0}+\frac{a_{+}^{\prime}(t)}{2 a(t)} \mu^{2}\left(1+\delta_{1}\right) y^{2}
\end{aligned}
$$




$$
\begin{aligned}
& +\frac{b_{+}^{\prime}(t)}{b(t)} V_{0}+\frac{C b_{+}^{\prime}(t)}{2 \nu\left(1-\delta_{0}\right) b(t)} y^{2}+\frac{\left|c^{\prime}(t)\right|}{\nu} y^{2} \\
& +\sqrt{2}(1+\mu)\left\{p_{1}(t)+p_{2}(t)\left(H(x)+y^{2}+z^{2}\right)^{\rho / 2}\right\}\left(y^{2}+z^{2}\right)^{1 / 2} \\
& +\sqrt{2} \Delta(1+\mu)\left(y^{2}+z^{2}\right) .
\end{aligned}
$$

Using the inequalities (4.4) and (4.7), and taking $\Delta<\min \left\{\frac{\mu b_{0} g_{0}-C h_{1}}{\sqrt{2}(1+\mu)}\right.$, $\left.\frac{a_{0} f_{0}-\mu}{\sqrt{2(1+\mu)}}\right\}$, we can find positive numbers $D_{4}$ and $D_{5}$ such that

$$
\begin{aligned}
\dot{V}_{0(4.1) \leqq} & -D_{4}\left(y^{2}+z^{2}\right)+D_{4}\left[a_{+}^{\prime}(t)+b_{+}^{\prime}(t)+\left|c^{\prime}(t)\right|\right] V_{0} \\
& +\sqrt{2}(1+\mu)\left\{p_{1}(t)+p_{2}(t)\right\}\left(y^{2}+z^{2}\right)^{1 / 2} \\
& +\sqrt{2}(1+\mu) p_{2}(t)\left(H(x)+y^{2}+z^{2}\right) .
\end{aligned}
$$

Now we define

$$
V(t, x, y, z)=e^{-\int_{0}^{t} \gamma(s) d s} \cdot V_{0}(t, x, y, z)
$$

where

$$
\gamma(s)=D_{5}\left(a_{+}^{\prime}(s)+b_{+}^{\prime}(s)+\left|c^{\prime}(s)\right|\right)+\frac{2 \sqrt{2}(1+\mu)}{D_{1}}\left\{p_{1}(s)+p_{2}(s)\right\}
$$

Then there exist two continuous functions $\tilde{w}_{1}(r), \tilde{w}_{2}(r)$ such that

$$
\tilde{w}_{1}(\|X\|) \leqq V(t, x, y, z) \leqq \tilde{w}_{2}((\|X\|)
$$

for all $X \in R^{3}$ and $t \in I$ where $\tilde{w}_{1}(r) \in C I P, \tilde{w}_{2}(r) \rightarrow \infty$ as $r \rightarrow \infty$ and $\tilde{w}_{2}(r)$ $\in C I$.

As in the Proof of Theorem 1,

$$
\begin{aligned}
\dot{V}_{(4.1)}= & e^{-\int_{0}^{t} \gamma(s) d s} \cdot\left[-D_{4}\left(y^{2}+z^{2}\right)+\sqrt{2}(1+\mu)\left\{p_{1}(t)+p_{1}(t)\right\}\left(y^{2}+z^{2}\right)^{1 / 2}\right. \\
& \left.-\sqrt{2}(1+\mu)\left\{p_{1}(t)+p_{2}(t)\right\}\left\{H(x)+y^{2}+z^{2}+2 k\right\}\right] \\
\leqq & e^{-\int_{0}^{t} \gamma(s) d s} \cdot\left[-D_{4}\left(y^{2}+z^{2}\right)\right.
\end{aligned}
$$




$$
\left.-\sqrt{2}(1+\mu)\left\{p_{1}(t)+p_{2}(t)\right\}\left(\sqrt{y^{2}+z^{2}}-\frac{1}{2}\right)^{2}-\frac{1}{4}+2 k\right] .
$$

Setting $k \geqq \frac{1}{8}$, we can find a positive number $D_{6}$ such that

$$
\dot{V}_{(4.1)} \leqq-D_{6}\left(y^{2}+z^{2}\right) .
$$

The remainder of the proof proceeds just as in the Proof of Theorem 1.

Q.E.D.

\section{Proof of Theorem 3}

In this section it will be assumed that $X=(x, y, z, w)$ and $\|X\|=$ $\sqrt{x^{2}+y^{2}+z^{2}+w^{2}}$.

The equation (1.4) is equivalent to the system

$(6.1)\left\{\begin{array}{l}\dot{x}=y \\ \dot{y}=z \\ \dot{z}=w \\ \dot{w}=-a(t) f(z) w-b(t) \phi(y, z)-c(t) g(y)-d(t) h(x)+p(t, x, y, z, w) .\end{array}\right.$

Our main tool is the function $V_{0}=V_{0}(t, x, y, z, w)$ defined by

$$
\begin{aligned}
2 V_{0}= & 2 \beta d(t) \int_{0}^{x} h(\xi) d \xi+2 c(t) \int_{0}^{y} g(\eta) d \eta+2 \alpha b(t) \int_{0}^{z} \phi(y, \zeta) d \zeta \\
& +2 a(t) \int_{0}^{z} f(\zeta) \zeta d \zeta+2 \beta a(t) y \int_{0}^{z} f(\zeta) d \zeta+\left\{\beta \phi_{0} b(t)-\alpha h_{0} d(t)\right\} y^{2} \\
& -\beta z^{2}+\alpha w^{2}+2 d(t) h(x) y+2 \alpha d(t) h(x) z+2 \alpha c(t) z g(y) \\
& +2 \beta y w+2 z w+k
\end{aligned}
$$

where $\alpha=\frac{1}{a_{0} f_{0}}+\varepsilon, \beta=\frac{h_{0} D}{c_{0} g_{0}}+\varepsilon$ and $\varepsilon, k$ are positive constants to be determined later in the proof. We have

$$
2 V_{0}=\frac{a(t)}{f_{1}(z)}\left\{\frac{w}{a(t)}+f_{1}(z) z+\beta f_{1}(z) y\right\}^{2}+2 \varepsilon d(t) \int_{0}^{x} h(\xi) d \xi
$$




$$
\begin{aligned}
& +2 d(t) \int_{0}^{x} h(\xi)\left[\frac{D h_{0}}{c_{0} g_{0}}-\frac{d(t) h^{\prime}(\xi)}{c(t) g_{1}(y)}\right] d \xi+c(t) \int_{0}^{y}\left\{g_{1}(\eta)-g^{\prime}(\eta)\right\} \eta d \eta \\
& +2 \alpha b(t) \int_{0}^{z}\left\{\phi(y, \zeta)-\phi_{0} \zeta\right\} d \zeta+\left\{\beta \phi_{0} b(t)-\alpha h_{0} d(t)-\beta^{2} a(t) f_{1}(z)\right\} y^{2} \\
& +a(t) \int_{0}^{z}\left\{f(\zeta)-f_{1}(\zeta)\right\} \zeta d \zeta+\left\{\alpha \phi_{0} b(t)-\beta-\alpha^{2} c(t) g_{1}(y)\right\} z^{2} \\
& +\left\{\alpha-\frac{1}{a(t) f_{1}(z)}\right\} w^{2}+\frac{c(t)}{g_{1}(y)}\left\{\frac{d(t)}{c(t)} h(x)+y g_{1}(y) z+\alpha g_{1}(y) z\right\}^{2}+k
\end{aligned}
$$

An elementary computation yields

$$
\begin{aligned}
& {\left[\frac{D h_{0}}{c_{0} g_{0}}-\frac{d(t) h^{\prime}(\xi)}{c(t) g_{1}(y)}\right] \geqq \frac{D h_{0} c_{0} g_{0}-c_{0} g_{0} D h^{\prime}(\xi)}{c_{0} c(t) g_{0} g_{1}(y)} \geqq 0,} \\
& 2 d(t) \int_{0}^{x} h(\xi)\left[\frac{D h_{0}}{c_{0} g_{0}}-\frac{d(t) h^{\prime}(\xi)}{c(t) g_{1}(y)}\right] d \xi \geqq 0, \\
& 2 \alpha b(t) \int_{0}^{z}\left\{\phi(y, t)-\phi_{0} \zeta\right\} d \zeta \geqq 0 .
\end{aligned}
$$

From the condition (vii), we have

$$
\frac{a_{0} b_{0} c_{0} f_{0} \phi_{0}}{C^{2}}>g^{\prime}(y), \frac{a_{0} b_{0} c_{0} \phi_{0} g_{0}}{A^{2} D h_{0}}>f(z),
$$

then

$$
\begin{aligned}
&\left\{\beta \phi_{0} b(t)-\alpha h_{0} d(t)-\beta^{2} a(t) f_{1}(z)\right\} \\
&= \beta\left\{\phi_{0} b(t)-\alpha c(t) g_{1}(y)-\beta a(t) f_{1}(z)\right\}+\alpha\left\{\beta c(t) g_{1}(y)-h_{0} d(t)\right\} \\
&= \beta\left\{\phi_{0} b(t)-\frac{c(t)}{a_{0} f_{0}} g^{\prime}(\tilde{y})-\frac{D h_{0}}{c_{0} g_{0}} a(t) f(\tilde{z})\right\}-\varepsilon \beta\left\{c(t) g^{\prime}(\tilde{y})+a(t) f(\tilde{z})\right\} \\
&+\alpha\left\{\beta c(t) g_{1}(y)-h_{0} d(t)\right\} \\
& \geqq \frac{\beta \delta_{0}}{a_{0} c_{0} f_{0} g_{0}}-\varepsilon \beta\left\{\frac{a_{0} b_{0} c_{0} f_{0} \phi_{0}}{C}+\frac{a_{0} b_{0} c_{0} \phi_{0} g_{0}}{A D h_{0}}\right\}+\alpha\left\{\left(\frac{h_{0} D}{c_{0} g_{0}}+\varepsilon\right) c_{0} g_{0}-h_{0} D\right\} \\
&= \frac{1}{A C a_{0} f_{0} c_{0}^{2} g_{0}^{2}}\left\{A C D h_{0} \delta_{0}-\varepsilon a_{0}^{2} b_{0} c_{0}^{2} f_{0} g_{0} \phi_{0}\left(A f_{0} D h_{0}+C g_{0}\right)\right\}
\end{aligned}
$$


$+\frac{\varepsilon}{A C D h_{0} a_{0} c_{0} f_{0} g_{0}}\left\{A C D h_{0} \delta_{0}-\varepsilon a_{0}^{2} b_{0} c_{0}^{2} f_{0} g_{0} \phi_{0}\left(A f_{0} D h_{0}+C g_{0}\right)\right\}+\varepsilon \alpha c_{0} g_{0}$.

If we take

$$
\varepsilon<\frac{A C D h_{0} \delta_{0}}{a_{0}^{2} b_{0} c_{0}^{2} f_{0} g_{0} \phi_{0}\left(A f_{0} D h_{0}+C g_{0}\right)},
$$

we have

$$
\begin{gathered}
\left\{\beta \phi_{0} b(t)-\alpha h_{0} d(t)-\beta^{2} a(t) f_{1}(z)\right\}>\frac{1}{A C a_{0} f_{0} c_{0}^{2} g_{0}^{2}}\left\{A C D h_{0} \delta_{0}\right. \\
\left.-\varepsilon a_{0}^{2} b_{0} c_{0}^{2} f_{0} g_{0} \phi_{0}\left(A D f_{0} h_{0}+C g_{0}\right)\right\} .
\end{gathered}
$$

Also using (6.4) we have

$$
\begin{aligned}
& \left\{\alpha \phi_{0} b(t)-\beta-\alpha^{2} c(t) g_{1}(y)\right\} \\
& \quad \geqq \frac{1}{C a_{0}^{2} A c_{0} D f_{0}^{2} g_{0} h_{0}}\left\{A C D h_{0} \delta_{0}-\varepsilon a_{0}^{2} b_{0} c_{0}^{2} f_{0} g_{0} \phi_{0}\left(A f_{0} D h_{0}+C g_{0}\right)\right\} \\
& \quad+\frac{\varepsilon}{a_{0} A C c_{0} D f_{0} g_{0} h_{0}}\left\{A C D h_{0} \delta_{0}-\varepsilon a_{0}^{2} b_{0} c_{0}^{2} f_{0} g_{0} \phi_{0}\left(A D f_{0} h_{0}+C g_{0}\right)\right\}+\varepsilon \beta a_{0} f_{0} .
\end{aligned}
$$

By (6.5), we have

$$
\begin{aligned}
& \left\{\alpha \phi_{0} b(t)-\beta-\alpha^{2} c(t) g_{1}(y)\right\} \\
& >\frac{1}{A C a_{0}^{2} f_{0}^{2} c_{0} g_{0} D h_{0}}\left\{A C D h_{0} \delta_{0}-\varepsilon a_{0}^{2} b_{0} c_{0}^{2} f_{0} g_{0} \phi_{0}\left(A f_{0} D h_{0}+C g_{0}\right)\right\}^{\bullet}
\end{aligned}
$$

Further,

$$
\begin{aligned}
& c(t) \int_{0}^{y}\left\{g_{1}(\eta)-g^{\prime}(\eta)\right\} \eta d \eta \geqq-\frac{C \delta}{2} y^{2}, \\
& a(t) \int_{0}^{z}\left\{f(\zeta)-f_{1}(\zeta)\right\} \zeta d \zeta \geqq-\frac{C c_{0} g_{0} \delta}{a_{0} f_{0} D h_{0}} \cdot \frac{z^{2}}{2}, \\
& \left\{\alpha-\frac{1}{a(t) f(z)}\right\} w^{2} \geqq \varepsilon w^{2} .
\end{aligned}
$$

Then we obtain 


$$
\begin{aligned}
2 V_{0} \geqq & 2 \varepsilon d_{0} \int_{0}^{x} h(\xi) d \xi \\
& +\frac{y^{2}}{2 A C a_{0} f_{0} c_{0}^{2} g_{0}^{2}}\left\{A C\left(2 D h_{0} \delta_{0}-C a_{0} f_{0} c_{0}^{2} g_{0}^{2} \delta\right)\right. \\
& \left.-2 \varepsilon a_{0}^{2} b_{0} c_{0}^{2} f_{0} g_{0} \phi_{0}\left(A f_{0} D h_{0}+C g_{0}\right)\right\} \\
& +\frac{z^{2}}{2 A C a_{0}^{2} f_{0}^{2} c_{0} g_{0} D h_{0}}\left\{A C\left(2 D h_{0} \delta_{0}-C a_{0} f_{0} c_{0}^{2} g_{0}^{2} \delta\right)\right. \\
& \left.-2 \varepsilon a_{0}^{2} b_{0} c_{0}^{2} f_{0} g_{0} \phi_{0}\left(A f_{0} D h_{0}+C g_{0}\right)\right\}+\varepsilon w^{2}+k .
\end{aligned}
$$

If we take

$$
\varepsilon<\frac{A C\left(2 D h_{0} \delta_{0}-C a_{0} f_{0} c_{0}^{2} g_{0}^{2} \delta\right)}{2 a_{0}^{2} b_{0} c_{0}^{2} f_{0} g_{0} \phi_{0}\left(A f_{0} D h_{0}+C g_{0}\right)}
$$

then there exists a positive number $D_{1}$ such that

$$
V_{0} \geqq D_{1}\left\{H(x)+y^{2}+z^{2}+w^{2}+k\right\} .
$$

From (6.4) it follows the boundedness of the functions $g_{1}(y)$ and $f_{1}(z)$, and we can see easily that there exists a positive number $D_{2}$ satisfying

$$
V_{0} \leqq D_{2}\left\{H(x)+y^{2}+z^{2}+w^{2}+k\right\}
$$

Therefore we have

$$
D_{1}\left(H(x)+y^{2}+z^{2}+w^{2}\right) \leqq V_{0} \leqq D_{2}\left(H(x)+y^{2}+z^{2}+w^{2}+k\right)
$$

Next along any solution $(x(t), y(t), z(t), w(t))$ of (6.1),

$$
\begin{aligned}
& 2 \dot{V}_{0(6.1)}=-2 \varepsilon c(t) g_{1}(y) y^{2}-2\left[\frac{h_{0} D}{c_{0} g_{0}} c(t) g_{1}(y)-d(t) h^{\prime}(x)\right] y^{2} \\
& -2[\alpha a(t) f(z)-1] w^{2}-2\left[\phi_{0} b(t)-\alpha c(t) g^{\prime}(y)-\beta a(t) f_{1}(z)\right] z^{2} \\
& -2 b(t)\left[\frac{\phi(y, z)}{z}-\phi_{0}\right]\left(z+\frac{\beta}{2} y\right)^{2}+\frac{\beta^{2}}{2} b(t)\left[\frac{\phi(y, z)}{z}-\phi_{0}\right] y^{2} \\
& \quad+2 \alpha b(t) z \int_{0}^{z} \phi_{y}(y, \zeta) d \zeta-2 \alpha d(t)\left[h_{0}-h^{\prime}(x)\right] y z
\end{aligned}
$$




$$
\begin{aligned}
& +2(\beta y+z+\alpha w) p(t, x, y, z, w)+2 \frac{\partial V_{0}}{\partial t} \\
& \leqq-2 \varepsilon c_{0} g_{0} y^{2}-2[\alpha a(t) f(z)-1] w^{2} \\
& -2\left[\phi_{0} b(t)-\alpha c(t) g^{\prime}(y)-\beta a(t) f_{1}(z)\right] z^{2}+\frac{\alpha^{2}}{2} d(t)\left[h_{0}-h^{\prime}(x)\right] z^{2} \\
& +\frac{\beta^{2}}{2} b(t)\left[\frac{\phi(y, z)}{z}-\phi_{0}\right] y^{2}+2(\beta y+z+\alpha w) p(t, x, y, z, w)+2 \frac{\partial V_{0}}{\partial t}
\end{aligned}
$$

If we take

$$
\begin{gathered}
\varepsilon_{0}<\varepsilon<\min \left\{\frac{A C\left(2 D h_{0} \delta_{0}-C a_{0} f_{0} c_{0}^{2} g_{0}^{2} \delta\right)}{2 a_{0}^{2} b_{0} c_{0}^{2} f_{0} g_{0} \phi_{0}\left(A f_{0} D h_{0}+C g_{0}\right)}, \frac{1}{a_{0} f_{0}}, \frac{D h_{0}}{c_{0} g_{0}},\right. \\
\left.\frac{A C D h_{0} \delta_{0}}{2 a_{0}^{2} b_{0} c_{0}^{2} f_{0} g_{0} \phi_{0}\left(A f_{0} D h_{0}+C g_{0}\right)}\right\},
\end{gathered}
$$

we can find a positive number $D_{3}$ such that

$$
\dot{V}_{0(6.1)} \leqq-2 D_{3}\left(y^{2}+z^{2}+w^{2}\right)+(\beta y+z+\alpha w) p(t, x, y, z, w)+\frac{\partial V_{0}}{\partial t}
$$

Let $D_{4}=\max (\alpha, \beta, 1)$, then

$$
\begin{aligned}
\dot{V}_{0(6.1)} \leqq & -2 D_{3}\left(y^{2}+z^{2}+w^{2}\right)+\sqrt{3} D_{4}\left(y^{2}+z^{2}+w^{2}\right)^{1 / 2}|p(t, x, y, z, w)|+\frac{\partial V_{0}}{\partial t} \\
\leqq & -2 D_{3}\left(y^{2}+z^{2}+w^{2}\right) \\
& +\sqrt{3} D_{4}\left(y^{2}+z^{2}+w^{2}\right)^{1 / 2}\left[p_{1}(t)+p_{2}(t)\left\{H(x)+y^{2}+z^{2}+w^{2}\right\} \rho / 2\right. \\
& \left.+\Delta\left(y^{2}+z^{2}+w^{2}\right)^{1 / 2}\right]+\frac{\partial V_{0}}{\partial t} .
\end{aligned}
$$

Taking $\Delta \leqq \frac{D_{3}}{\sqrt{3} D_{4}}$, we have

$$
\begin{aligned}
& \dot{V}_{0(6.1)} \leqq-D_{3}\left(y^{2}+z^{2}+w^{2}\right) \\
& \quad+\sqrt{3} D_{4}\left(y^{2}+z^{2}+w^{2}\right)^{1 / 2}\left[p_{1}(t)+p_{2}(t)\left\{H(x)+y^{2}+z^{2}+w^{2}\right\}^{\rho / 2}\right] \\
& \quad+\frac{\partial V_{0}}{\partial t}
\end{aligned}
$$


From the assumptions in Theorem 3 and (6.4) we have a positive number $D_{5}$ satisfying

$$
\frac{\partial V_{0}}{\partial t} \leqq D_{5}\left\{\left|a^{\prime}(t)\right|+b_{+}^{\prime}(t)+\left|c^{\prime}(t)\right|+\left|d^{\prime}(t)\right|\right\} \cdot V_{0} .
$$

Note that

$$
\left(H(x)+y^{2}+z^{2}+w^{2}\right)^{\rho / 2} \leqq 1+\left(H(x)+y^{2}+z^{2}+w^{2}\right)^{1 / 2} .
$$

(6.10), (6.11) and (6.12) show that

$$
\begin{aligned}
& \dot{V}_{0(6.1)} \leqq-D_{3}\left(y^{2}+z^{2}+w^{2}\right) \\
& \quad+D_{5}\left\{\left|a^{\prime}(t)\right|+b_{+}^{\prime}(t)+\left|c^{\prime}(t)\right|+\left|d^{\prime}(t)\right|\right\} \cdot V_{0} \\
& +\sqrt{3} D_{4}\left\{p_{1}(t)+p_{2}(t)\right\}\left(y^{2}+z^{2}+w^{2}\right)^{1 / 2} \\
& \quad+\sqrt{3} D_{4} p_{2}(t)\left(H(x)+y^{2}+z^{2}+w^{2}\right) .
\end{aligned}
$$

Now we define

$$
V(t, x, y, z, w)=e^{-\int_{0}^{t} \gamma(s) d s} \cdot V_{0}(t, x, y, z, w)
$$

where

$$
\gamma(s)=D_{5}\left(\left|a^{\prime}(s)\right|+b_{+}^{\prime}(s)+\left|c^{\prime}(s)\right|+\left|d^{\prime}(s)\right|\right)+\frac{2 \sqrt{3} D_{4}}{D_{1}}\left\{p_{1}(s)+p_{2}(s)\right\} .
$$

Then it is easy to see that there exist two continuous functions $w_{1}(r)$, $w_{2}(r)$ satisfying

$$
w_{1}(\|X\|) \leqq V(t, x, y, z, w) \leqq w_{2}(\|X\|)
$$

for all $X \in R^{4}$ and $t \in I$ where $w_{1}(r) \in C I P, w_{1}(r) \rightarrow \infty$ as $r \rightarrow \infty$ and $w_{2}(r)$ $\in C I$.

Along any solution $(x(t), y(t), z(t), w(t))$ of $(6.1)$ we have

$$
\begin{aligned}
\dot{V}_{(6.1)} & =e^{-\int_{0}^{t} \gamma(s) d s} \cdot\left[\dot{V}_{0(6.1)}-\gamma(t) V_{0}\right] \\
& \leqq e^{-\int_{0}^{1} \gamma(s) d s} \cdot\left[-D_{3}\left(y^{2}+z^{2}+w^{2}\right)\right.
\end{aligned}
$$




$$
\begin{aligned}
& +\sqrt{3} D_{4}\left\{p_{1}(t)+p_{2}(t)\right\}\left\{\left(y^{2}+z^{2}+w^{2}\right)^{1 / 2}\right. \\
& \left.-\sqrt{3} D_{4}\left\{p_{1}(t)+p_{2}(t)\right\}\left\{H(x)+y^{2}+z^{2}+w^{2}+2 k\right\}\right] \\
& \leqq \\
& e^{-\int_{0}^{t} \gamma(s) d s} \cdot\left[-D_{3}\left(y^{2}+z^{2}+w^{2}\right)\right. \\
& \left.-\sqrt{3} D_{4}\left\{p_{1}(t)+p_{2}(t)\right\}\left\{\left(\sqrt{y^{2}+z^{2}+w^{2}}-\frac{1}{2}\right)^{2}-\frac{1}{4}+2 k\right\}\right] .
\end{aligned}
$$

Setting $k \geqq \frac{1}{8}$, we can find a positive number $D_{6}$ such that

$$
\dot{V}_{(6.1)} \leqq-D_{6}\left(y^{2}+z^{2}+w^{2}\right) .
$$

From the inequalities (6.15) and (6.16), we obtain the uniform boundedness of all the solutions $(x(t), y(t), z(t), w(t))$ of $(6.1)$.

In the system (6.1) we set

$$
\begin{aligned}
& F(t, X)=\left(\begin{array}{c}
x \\
y \\
z \\
-a(t) f(z) w-b(t) \phi(y, z)-c(t) g(y)-d(t) h(x)
\end{array}\right), \\
& G(t, X)=\left(\begin{array}{c}
0 \\
0 \\
0 \\
p(t, x, y, z, w)
\end{array}\right),
\end{aligned}
$$

then

$$
\|G(t, X)\| \leqq p_{1}(t)+p_{2}(t)\left\{H(x)+y^{2}+z^{2}+w^{2}\right\}^{\rho / 2}+\Delta\left(y^{2}+z^{2}+w^{2}\right)^{1 / 2} .
$$

Let

$$
G_{1}(t, X)=p_{1}(t)+p_{2}(t)\left\{H(x)+y^{2}+z^{2}+w^{2}\right\}^{\rho / 2}
$$

and

$$
G_{2}(X)=\Delta\left(y^{2}+z^{2}+w^{2}\right)^{1 / 2}
$$


It is clear that $F(t, X)$ and $G_{1}(t, X)$ satisfy the conditions of Lemma 2 .

Let $W(X)=D_{6}\left(y^{2}+z^{2}+w^{2}\right)$, then

$$
\dot{V}_{(6.1)}(t, x, y, z, w) \leqq-W(X)
$$

and $W(X)$ is positive definite with respect to the closed set $\Omega \equiv\{(x, y, z, w) \mid$ $\left.x \in R^{1}, y=0, z=0, w=0\right\}$.

It follows that on $\Omega$

$$
F(t, X)=\left(\begin{array}{c}
0 \\
0 \\
0 \\
-d(t) h(x)
\end{array}\right)
$$

By the conditions (i) and ( $\mathrm{x}$ ), we have $d(t) \rightarrow d_{\infty}$ as $t \rightarrow \infty$ where $0<d_{0} \leqq d_{\infty} \leqq D$. It is also clear that if we take

$$
\tilde{H}(X) \equiv\left(\begin{array}{c}
0 \\
0 \\
0 \\
-d_{\infty} h(x)
\end{array}\right),
$$

then the conditions (a) and (b) of Lemma 2 are satisfied.

Moreover $G_{2}(X)$ is positive definite with respect to the closed set $\Omega$ and the condition (c) of Lemma 2 is satisfied.

The remainder of the proof is analogous to that of Theorem 1 .

Q.E.D.

\section{References}

[1] Asmussen, M.A., On the behavior of solutions of certain differential equations of the fourth order, Ann. Mat. Pura. Appl., 89 (1971), 121-143.

[2] Cartwright, M.L., On the stability of solutions of certain differential equations of the fourth order, Quart. J. Mech. Appl. Math., 9 (1956), 185-194.

[3] Ezeilo, J.O.C., On the boundedness and the stability of solutions of some differential equations of the fourth order, J. Math. Anal. Appl., 5 (1962), 136-146.

[4] Stability results for the solutions of some third and fourth order differ- 
ential equations, Ann. Mat. Pura. Appl., 66 (1964), 233-249.

[5] Hara, T., On the stability of solutions of certain third order differential equations, Proc. Japan Acad., 47 (1971), 897-902

[6] - On the asymptotic behavior of solutions of certain third order ordinary differential equations, Proc. Japan. Acad., 47 (1971), 903-908.

[7] A remark on the asymptotic behavior of the solution of $\dddot{x}+f(\ddot{x}) \dddot{x}$ $+\phi(\dot{x}, \ddot{x})+g(\dot{x})+\mathrm{h}(x)=\mathrm{p}(t, x, \dot{x}, \ddot{x}, \dddot{x})$, Proc. Japan Acad., 48 (1972), 353-355.

[8] - Remarks on the asymptotic behavior of the solutions of certain nonautonomous differential equations, Proc. Japan Acad., 48 (1972), 549-552.

[9] - On the asymptotic behavior of solutions of certain non-autonomous differential equations, to appear in Osaka J. Math.

[10] Harrow, M., A stability result for solutions of certain fourth order homogeneous differential equations, J. London Math. Soc., 42 (1967), 51-56.

[11] - Further results on the boundedness and the stability of solutions of some differential equations of the fourth order. SIAM J. Math. Anal., 1 (1970), 189-194.

[12] Lalli, B.S. and Skrapek, W.S., On the boundedness and stability of some differential equations of the fourth order, SIAM J. Math. Anal., 2 (1971), 221-225.

[13] - Some further stability and boundedness results of some differential equations of the fourth order. Ann. Mat. Pura. Appl., 90 (1971), 167-179.

[14] Reissig, R., Sansone, G. und Conti, R., Nichtlineare Differentialgleichungen Höherer Ordnung, Consiglio Nazionale delle Ricerche, Monografie Matematiche. No. 16 (1969).

[15] Sinha, A.S.C., and Hoft, R.G., Stability of a nonautonomous differential equation of fourth order, SIAM J. Control, 9 (1971), 8-14.

[16] Swick, K.E., On the boundedness and the stability of solutions of some nonautonomous differential equations of the third order, J. London Math. Soc., 44 (1969), 347-359.

[17] — Asymptotic behavior of the solutions of certain third order differential equations, SIAM J. Appl. Math., 19 (1970), 96-102.

[18] Tejumola, H.O., A note on the boundedness and the stability of solutions of certain third-order differential equations, Ann. Mat. Pura. Appl., 92 (1972) 6575.

[19] Yamamoto, M., On the stability of the solutions of some non-autonomous differential equations of the third order, Proc. Japan Acad., 47 (1971), 909-914.

[20] - Remarks on the asymptotic behavior of the solutions of certain third order non-autonomous differential equations, Proc. Japan Acad., 47 (1971), 915920.

[21] Yoshizawa, T., Stability Theory by Liapunov's Second Method, The Mathematical Society of Japan, 1966.

[22] Zarghamee, M.S., and Mehri, B., On the behavior of solutions of certain third order differential equations, J. London Math. Soc., (2), 4 (1971), 271-276. 
